Скопје, Македонија

\title{
BARZILAI-BORWEIN METHOD FOR A NONLOCAL ELLIPTIC PROBLEM
}

\author{
MIROSLAV S. PETROV AND TODOR D. TODOROV
}

\begin{abstract}
The object of interest in the present paper is a nonlocal nonlinear problem for a general second order elliptic operator. The problem under consideration represents a model of nonlocal reaction diffusion process. Furthermore, applications in computational biology are also available. The strong problem is reduced to a discrete minimization problem. The approximate problem is obtained by Lagrangian finite element discretizations. Due to its simplicity and efficiency, the Barzilai and Borwein gradient method is used for finding positive solutions with respect to the inhomogeneous strong Allee effect growth pattern. The corresponding fast and stable iterative algorithm converges monotonically with respect to the objective functional. A rigorous proof of the monotone convergence theorem is presented. Computer implementations of the method support the considered theory.
\end{abstract}

\section{INTRODUCTION}

The population growth is more than eighty millions annually [1]. The human population increases by $1.2 \%$ each year. The global population has grown from one billion in year 1800 to seven billion in year 2012 [1]. There is a real chance it reaches more than eleven billion by the end of the century. The exceeding of the resource capacity of an area or environment is called overpopulation. It affects directly the growth of the population. The overpopulation problem is very important from economical and social point of view. The mathematical model of population behavior consists of the nonlinear elliptic problem

$$
\frac{\partial u}{\partial t}=\mathcal{D} \Delta u+u f(x, u)
$$

defined on a bounded polygonal domain. The solution $u$ describes the population density, $\mathcal{D}>0$ denotes the diffusion constant, $\Delta$ is the spatial Laplacian and $f(x, u)$ is the growth rate per capita. If the function $f(x, u)$ is negative when $u$ is small, we call that a strong Allee effect is available [2]. Otherwise if $f(x, u)$ is smaller than the maximum but still positive for small $\mathrm{u}$, we call it a weak Allee effect [2]. The application of the reaction-diffusion equation in computational

2010 Mathematics Subject Classification. 65N30, 65K10, 90C06.

Key words and phrases. Nonlocal nonlinear problem, Kirchhoff type equation, BarzilaiBorwein iterative method, finite element approximations. 
biology is studied by a lot of researchers in the last decades [2]-[5]. A nonlocal reaction-diffusion model

$$
\begin{aligned}
& \frac{\partial u}{\partial t}-a\left(\int_{\Omega}|\nabla u|^{2}\right) \Delta u=f(x, u), \\
& a \in C\left(\mathbb{R}^{+}\right), \quad 0<m \leq a(p) \leq M
\end{aligned}
$$

is studied by M. Chipot, V. Valente, G. V. Caffarelli [6]. More general reactiondiffusion equation is considered by S. A. Sanni [7].

The object of interest in the present paper is the nonlocal nonlinear reactiondiffusion problem investigated by T. D. Todorov [8]. The investigation is carried out in the case of a general second-order elliptic operator. The nonlocal term involved in the strong formulation essentially increases the complexity of the problem and the necessary total computational work. The major contribution of the present paper consists of a monotone convergent iterative method for solving the nonlocal nonlinear elliptic problem. A new globalization technique with variable steplength is obtained. A convergence theorem is proved. The new method is computer implemented.

The rest of the article is organized as follows. The problem under consideration is described in Section 2. The weak formulation and discrete problem are obtained in Section 3. An iterative schemes for solving the nonlocal elliptic problem is compiled in Section 4. The corresponding algorithm is described briefly step by step. Section 5 contains some numerical results supporting the considered theory. Concluding remarks are involved in Section 6.

\section{Problem Definition}

Let $\Omega$ be a plane polygon. Denote the norm and the seminorm in the real Sobolev space $H^{k}(\Omega)$ by $\|\cdot\|_{k, \Omega}$ and the $|\cdot|_{k, \Omega}$. Introduce the norm

$$
\left\|D^{k} F(x)\right\|=\sup _{\substack{\left\|\xi_{i}\right\| \leq 1 \\ 1 \leq i \leq k}}\left\|D^{k} F(x)\left(\xi_{1}, \xi_{2}, \ldots, \xi_{k}\right)\right\|
$$

for the $k$-th Fréchet derivative $D^{k} F(x)$. The $L^{2}$-scalar product is denoted by

$$
(u, v)=\int_{\Omega} u v d x
$$

Define the space

$$
\mathbf{V}=\left\{v \in H^{1}(\Omega) \mid v=0 \text { on } \Gamma\right\} .
$$

and the second-order elliptic linear operator

$$
L u=-\sum_{i, j=1}^{2} \frac{\partial}{\partial x_{j}}\left(a_{i j} \frac{\partial u}{\partial x_{i}}\right)+a . u, \operatorname{dom} L=C_{0}^{2}(\bar{\Omega}),
$$

where $a_{i j}(x)$ and $a(x)$ belong to $C^{1}(\bar{\Omega}), a_{i j}=a_{j i}, i, j=1,2$ and $a(x) \geq a_{0}>$ $0, \forall x \in \Omega$. Assume that $L$ is uniformly elliptic, i.e. there exists a constant $\alpha>0$ 
such that

$$
\alpha \sum_{i=1}^{n} \xi_{i}^{2} \leq \sum_{i, j=1}^{2} a_{i j}(x) \xi_{i} \xi_{j}, \quad \forall \xi, x \in \mathbb{R}^{2} .
$$

Suppose that:

$$
\begin{gathered}
g \in C^{1}(\bar{\Omega} \times \mathbb{R}), \quad \frac{\partial g}{\partial u}(x, u) \geq 0, \quad \forall x \in \bar{\Omega}, \quad g(x, 0)=0, \\
f \in L^{2}(\Omega) \text { with }\|f\|_{0, \Omega} \neq 0 .
\end{gathered}
$$

Consider the following nonlocal nonlinear elliptic problem

$$
\mathcal{S}:\left\{\begin{array}{c}
\text { Find } u \in C_{0}^{2}(\bar{\Omega}) \text { satisfying : } \\
(L u, u) L u+g(x, u)=f(x) \text { in } \Omega, . \\
u=0 \text { on } \partial \Omega
\end{array} .\right.
$$

\section{THE WEAK FORMULATION AND DISCRETIZATION}

Applying Green's theorem to $(\mathcal{S})$ we obtain the weak formulation

$$
\mathcal{W}:\left\{\begin{array}{c}
\text { Find } u \in \mathbf{V} \text { such that } \\
a(u, u) a(u, v)+(g(x, u), v)=(f, v) \quad \text { in } \quad \Omega,
\end{array}\right.
$$

where $a(u, v)$ is the bilinear form

$$
a(u, v)=\int_{\Omega} \sum_{i, j=1}^{2} a_{i j}(x) \frac{\partial u}{\partial x_{i}} \frac{\partial v}{\partial x_{j}} d x+\int_{\Omega} a(x) u v d x .
$$

and $(\cdot, \cdot)$ is the $L^{2}$-scalar product. Since $L$ is a linear continuous and uniformly V-elliptic operator there exist positive constants $\underline{\alpha}$ and $\bar{\alpha}$ such that

$$
\underline{\alpha}\|u\|_{1, \Omega}^{2} \leq a(u, u), \quad a(u, v) \leq \bar{\alpha}\|u\|_{1, \Omega}\|v\|_{1, \Omega}, \quad \forall u, v \in \mathbf{V} .
$$

Define the objective functional

$$
J(v)=\frac{a^{2}(v, v)}{4}+B(v)-F(v),
$$

where

$$
G(v)=\int_{0}^{v} g(x, t) d t, \quad F(v)=(f, v) \text { and } B(v)=\int_{\Omega} G(v) d x .
$$

Associate the weak form $(\mathcal{W})$ with the following minimization problem

$$
\mathcal{M}: \arg \min _{v \in \mathbf{V}} J(v) .
$$

The existence and uniqueness of the solution of the minimization problem $(\mathcal{M})$ is established in [8, Theorem 1].

Continue with discretization and formulation of the approximate finite element problem. Let $\left\{\tau_{h}\right\}$ be a regular family of conforming finite element triangulations obtained by Lagrangian finite elements of degree $n, \mathbf{V}_{h} \subset \mathbf{V}$ be the corresponding finite element space, $\mathcal{N}_{h}=\left\{a_{i}\right\}_{i=1}^{m}$ be the set of all interior nodes of $\tau_{h}$ and 
$\left\{\varphi_{i}(x)\right\}_{i=1}^{m}$ be the nodal bases functions of $\mathbf{V}_{h}$. Consider the following discrete problem

$$
\mathcal{W}_{h}:\left\{\begin{array}{c}
\text { Find a function } u_{h} \in \mathbf{V}_{h} \text { satisfying } \\
a\left(u_{h}, u_{h}\right) a\left(u_{h}, v_{h}\right)+\left(g\left(x, u_{h}\right), v_{h}\right)=\left(f, v_{h}\right), \quad \forall v_{h} \in \mathbf{V}_{h}
\end{array} .\right.
$$

The finite element solution can be presented in the form

$$
u_{h}(x)=U \cdot \Phi(x)=\sum_{i=1}^{m} U_{i} \varphi_{i}(x) \in \mathbf{V}_{h},
$$

where $U_{i}=u_{h}\left(a_{i}\right)$. As in [8] we interpolate the function $g$ by a $\mathbf{V}_{h}$ interpolant

$$
I_{h} g=\sum_{i=1}^{m} g\left(a_{i}, u\left(a_{i}\right)\right) \varphi_{i} .
$$

Compile the quartic problem

$$
\hat{\mathcal{Q}}_{h}:\left\{\begin{array}{l}
\text { Find a function } u_{h} \in \mathbf{V}_{h} \text { that satisfies } \\
a\left(\hat{u}_{h}, \hat{u}_{h}\right) a\left(\hat{u}_{h}, v_{h}\right)+\left(g_{h}, v_{h}\right)=\left(f, v_{h}\right), \quad \forall v_{h} \in \mathbf{V}_{h}
\end{array},\right.
$$

where $g_{h}=I_{h} g$. Define

$$
G_{h}(v)=\int_{0}^{v} g_{h}(x, t) d t, \quad B_{h}(v)=\int_{\Omega} G_{h}(v) d x
$$

and corresponding objective functionals:

$$
\begin{gathered}
J\left(v_{h}\right)=\frac{1}{4} a^{2}\left(v_{h}, v_{h}\right)+B\left(v_{h}\right)-F\left(v_{h}\right), \quad v_{h} \in \mathbf{V}_{h}, \\
J_{h}\left(v_{h}\right)=\frac{1}{4} a^{2}\left(v_{h}, v_{h}\right)+B_{h}\left(v_{h}\right)-F\left(v_{h}\right), \quad v_{h} \in \mathbf{V}_{h}
\end{gathered}
$$

for the problems $\left(\mathcal{W}_{h}\right)$ and $\left(\hat{\mathcal{Q}}_{h}\right)$.

Remark that

$$
D J_{h}\left(\hat{u}_{h}\right) v_{h}=a\left(\hat{u}_{h}, \hat{u}_{h}\right) a\left(\hat{u}_{h}, v_{h}\right)+\left(g_{h}, v_{h}\right)-\left(f, v_{h}\right), \quad \forall v_{h} \in \mathbf{V}_{h} .
$$

4. A globalization technique With Variable Steplength For SOlving THE DISCRETE PROBLEM

Consider the following unconstraint minimization problem

$$
\mathcal{M}_{h}: \arg \min _{v \in \mathbf{V}_{h}} J(v) .
$$

Define the Barzilai-Borwein gradient method

$$
\left(u_{k+1}, v\right)=\left(u_{k}, v\right)-\frac{1}{\alpha_{k}} D J\left(u_{k}\right) v, \quad k \geq 1
$$

with the steplength

$$
\begin{gathered}
\alpha_{k}=\frac{\left(a\left(u_{k}, u_{k}\right)+a\left(u_{k-1}, u_{k-1}\right)\right) a\left(s_{k-1}, s_{k-1}\right)+\left(\left(g\left(x, u_{k}\right)+g\left(x, u_{k-1}\right)\right) s_{k-1}, s_{k-1}\right)}{2\left\|s_{k-1}\right\|_{1, \Omega}^{2}} \\
s_{k-1}=u_{k}-u_{k-1}
\end{gathered}
$$


obtained by T. D. Todorov [8]. He proved that the steplength (4.2) produces much better results in the quartic case than the classical steplength

$$
\beta_{k}=\frac{D F\left(u_{k}\right) s_{k-1}}{\left\|s_{k-1}\right\|_{1, \Omega}^{2}}
$$

analyzed by E. G. Birgin, J. M. Martínez and M. Raydan [9]. In the proof of [8, Theorem 2] was established that

$$
0<\alpha_{*}<\alpha_{k}<\alpha^{*}, \quad \forall k \in \mathbb{N},
$$

where $\alpha_{*}$ and $\alpha^{*}$ are positive constants. The validity of (4.4) depends on the distance between the initial guesses and the weak solution. If the initial guesses are faraway from the weak solution $\alpha_{k}$ could become negative for some $k$. That is why in such cases we cannot guarantee monotone convergence of the objective functional. Moreover the lack of estimate (4.4) can lead to divergence of the two point step size gradient method (4.1-4.2).

In this section we propose a monotone convergent algorithm for the two point gradient method in the quartic case.

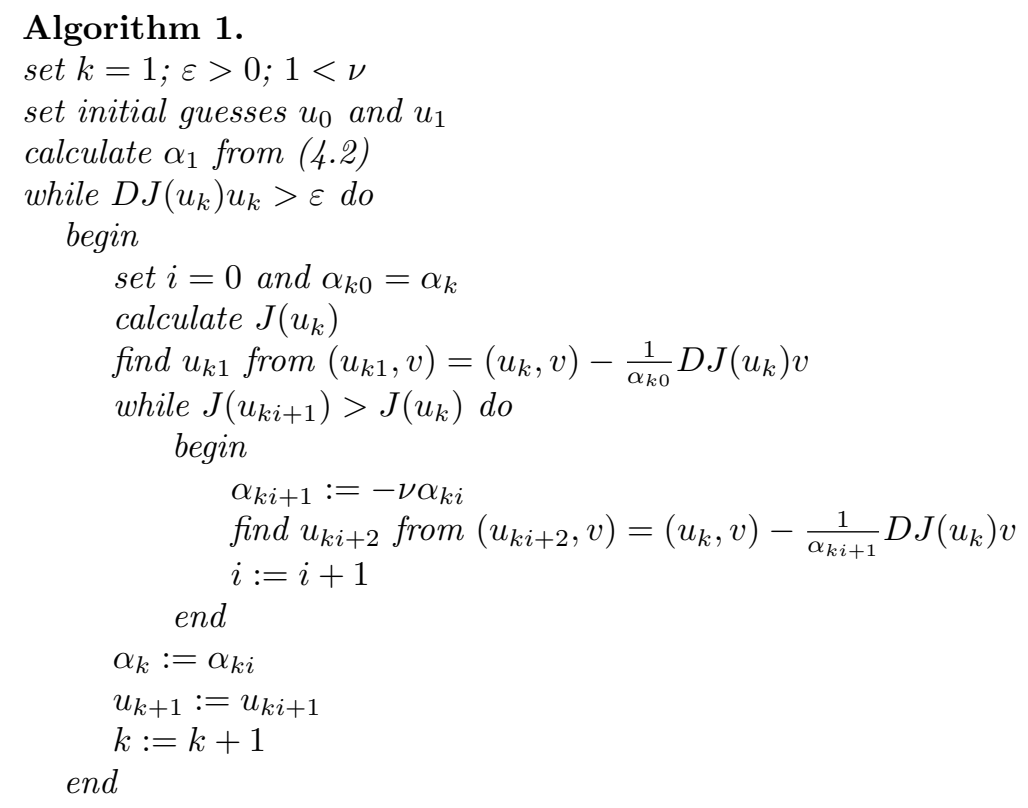

Theorem 1. Assume that conditions (2.1-2.3) hold. Then the sequence $\left\{J\left(u_{k}\right)\right\}$ generated by Algorithm 1 converges monotonically to $\left\{J\left(u_{h}\right)\right\}$.

Proof. Compare two successive values of the objective functional. Apply the mean value theorem

$$
J\left(u_{k i+1}\right)-J\left(u_{k}\right)=D J\left(\widetilde{u}_{k i+1}\right)\left(u_{k i+1}-u_{k}\right),
$$


where $\widetilde{u}_{k i+1}=u_{k}+\vartheta\left(u_{k i+1}-u_{k}\right), \quad \vartheta \in(0,1)$. From the definition of $u_{k i+1}$ we have

$$
J\left(u_{k i+1}\right)-J\left(u_{k}\right)=-\frac{1}{\alpha_{k i}} D J\left(\widetilde{u}_{k i+1}\right) D J\left(u_{k}\right) .
$$

Remember that the functional $J(u)$ is twice Fréchet differentiable. Therefore, from the sign preservation property, there is a neighborhood

$$
U_{\varepsilon}\left(u_{k}\right)=\left\{v \in \mathbf{V}_{h} \mid\left\|u_{k}-v\right\|_{1, \Omega}<\varepsilon\right\}
$$

such that $D J(v) D J\left(u_{k}\right)>0 \forall v \in U_{\varepsilon}\left(u_{k}\right)$. We have $\left\{\begin{array}{l}u_{k i} \rightarrow u_{k} \\ i \rightarrow+\infty\end{array}\right.$

since $\left\{\begin{array}{l}\left|\alpha_{k i}\right| \rightarrow+\infty \\ i \rightarrow+\infty\end{array}\right.$. Then there is an integer $i_{0}$ such that

$$
u_{k i+1} \in U_{\varepsilon}\left(u_{k}\right) \forall i \geq i_{0} .
$$

Let $\underline{i}$ be the smallest $i$ satisfying (4.5) and $\alpha_{k i}>0$. Then

$$
J\left(u_{k \underline{i}+1}\right)-J\left(u_{k}\right)=-\frac{1}{\alpha_{k \underline{i}}} D J\left(\widetilde{u}_{k \underline{i}}\right) D J\left(u_{k}\right)<0 .
$$

We choose $u_{k+1}=u_{k i+1}$ and obtain $J\left(u_{k+1}\right)<J\left(u_{k}\right)$. Thus we proved that the sequence $\left\{J\left(u_{k}\right)\right\}$ is monotone decreasing. Since $J\left(u_{k}\right) \geq J\left(u_{h}\right)[8]$ we conclude that $\left\{J\left(u_{k}\right)\right\}$ converges monotonically.

It remains to prove that

$$
\lim _{k \rightarrow+\infty} J\left(u_{k}\right)=J\left(u_{h}\right) .
$$

Suppose the negation of (4.6) namely $\left\{\begin{array}{l}u_{k} \rightarrow u_{\infty} \\ k \rightarrow+\infty\end{array}\right.$ and $\left\|u_{\infty}-u_{h}\right\|_{1, \Omega_{h}} \neq 0$. Then

$$
J\left(u_{\infty}\right)=\inf _{k \in \mathbf{N}} J\left(u_{k}\right)
$$

Choosing a sufficiently large $\alpha_{\infty}>0$ we obtain

$$
u_{*}=u_{\infty}-\frac{1}{\alpha_{\infty}} D J\left(u_{\infty}\right)
$$

and $J\left(u_{*}\right)<J\left(u_{\infty}\right)$. The last result contradicts to (4.7). Therefore (4.6) is valid which completes the proof.

\section{Numerical Tests}

Consider an initial uniform triangulation of the unit square $\Omega$ by 18 cubic triangular Lagrangian finite elements. Obtain triangulations with 162 and 1458 applying the Jung and Todorov [10] refinement strategy. All numerical tests are executed by Andreev and Todorov [11] cubature formula of degree five. This cubature formula is appropriate for obtaining optimal convergence properties with respect to the cubic Lagrangian finite element.

Denote the error functional by

$$
\mathcal{E}(v)=\left|D J_{h}(v) v\right|
$$

and $\varepsilon=10^{-6}$. 


\begin{tabular}{||r|r|r|r|r|l||}
\hline \hline$F E$ & $\operatorname{dim} \mathbf{V}_{h}$ & $\widetilde{\alpha}_{k}$ & $\hat{\alpha}_{k}$ & $\hat{\beta}_{k}$ & $\nu_{k}$ \\
\hline 18 & 64 & 17 & 39 & 45 & 1.5 \\
162 & 676 & 68 & 168 & 179 & 2 \\
1458 & 6400 & 272 & 631 & 852 & 4 \\
\hline \hline
\end{tabular}

TABLE 1. The number of necessary iterations solving Problem 1 to obtain $\mathcal{E}\left(\hat{u}_{k}\right)<\varepsilon$.

Definition 1. The function $i_{h} \in \mathbf{V}_{h}$ is said to be quasiunit if $i_{h}(x)=1 \forall x \in \mathcal{N}_{h}$.

Problem 1. State the problem

$$
\left\{\begin{array}{c}
(L u, u) L u+g(u)=f(x) \text { in } \Omega, \\
u=0 \text { on } \partial \Omega
\end{array}\right.
$$

where $L=-\triangle, g(u)=u\left(2 u^{2}-3 u+6\right)$ and $f(x)=\mathrm{e}^{x_{1} x_{2}}$.

The function $-\frac{g(u)}{u}$ satisfies the inhomogeneous strong Allee effect growth pattern. Denote the steplength obtained in Algorithm 1 by $\widetilde{\alpha}_{k}$, the steplength obtained by T. D. Todorov [8] by $\hat{\alpha}_{k}$, the analogue of (4.3) related to the quartic problem by $\hat{\beta}$. Solve Problem 1 with initial guesses

$$
\hat{u}_{0}=4 i_{h} \text { and } \hat{u}_{1}=\frac{1}{4} i_{h} .
$$

The optimal values of the parameter $\nu$ forms an increasing sequence $\left\{\nu_{k}\right\}$ depending on the refinement level. Results are presented in comparative Table 1. The presented numerical examples indicate that the proposed monotone BarzilaiBorwein method is superior with respect to the number of iterations.

\section{Conclusion}

A nonlocal nonlinear problem for a general second order elliptic operator is reduced to a quartic minimization problem. An effective monotone convergent algorithm for solving the problem of interest is obtained. A rigorous proof of the convergence theorem is presented. The numerical tests indicate that the number of necessary iterations for satisfying the stop criterion are essentially reduced.

\section{REFERENCES}

[1] World Population estimates by the US Census Bureau, USCB. Retrieved May 22, 2012.

[2] J. Shi, R. Shivaji, Persistence in reaction diffusion models with weak allee effect, Mathematical Biology, J. Math. Biol. 52, (2006) 807-829.

[3] C. O. Alves, F.J.S. Correa, G.M. Figueiredo, On a class of nonlocal elliptic problems with critical growth, Differential Equations and Applications 2, (2010) 409-417.

[4] R. S. Cantrell, C. Cosner, Spatial ecology via reaction-diffusion equation, Wiley series in mathematical and computational biology, John Wiley \& Sons, Ltd, 2004.

[5] G. Liu, Y. Wang, J. Shi, Existence and nonexistence of positive solutions of semilinear elliptic equation with inhomogeneous strong Allee effect, Applied Mathematics and Mechanics, English Edition 30, (2009) 1461-1468. 
[6] M. Chipot, V. Valente, G.V. Caffarelli, Remarks on a nonlocal problem involving the Dirichlet energy, Rendiconti del Seminario Matematico della universitaÂădi Padova 110, (2003) 199-220.

[7] S. A. Sanni, Nonlocal degenerate reaction-diffusion equations with general nonlinear diffusion term, Electronic Journal of Differential Equations, vol. 2014, 124 (2014), 1-27.

[8] T. D. Todorov, A nonlocal problem for a general second-order elliptic operator, Computers \& Mathematics with Applications, vol.69, issue 5, (2015), 411-422.

[9] E. G. Birgin, J. M. Martínez, M. Raydan, Spectral Projected Gradient methods: Review and Perspectives, Journal of Statistical Software, vol. 60, 3, 2014.

[10] M. Jung and T. D. Todorov, Isoparametric multigrid method for reaction-diffusion equations, Applied Numerical Mathematics, 56, (2006) 1570-1583.

[11] A. B. Andreev, T. D. Todorov, Isoparametric numerical integration on triangular finite element meshes, Comptes rendus de l'Academie bulgare des Sciences, 17, vol. 57, (2004) $37-44$.

Department of Technical Mechanics, Technical University, 5300 Gabrovo, Bulgaria

E-mail address: mirspetrov@gmail.com

Department of Mathematics, Technical University,

5300 Gabrovo, Bulgaria

E-mail address: t.todorov@yahoo.com 